\title{
What you always wanted to know about Huntington's disease
}

$\bullet \bullet \bullet \bullet \bullet \bullet \bullet \bullet \bullet \bullet \bullet \bullet \bullet \bullet \bullet \bullet \bullet \bullet \bullet \bullet \bullet \bullet \bullet \bullet \bullet \bullet \bullet \bullet \bullet \bullet \bullet \bullet \bullet \bullet \bullet \bullet \bullet \bullet \bullet \bullet \bullet \bullet \bullet \bullet \bullet$

Questions and answers about HD

'Huntington's Disease' (second edition)

by Oliver Quarrell, thefacts series, Oxford University Press, 2008

ISBN 978-0-19-921201-9 (Pbk)

$\ldots \ldots \ldots$

Gerry Evers-Kiebooms

European Journal of Human Genetics (2010) 18, 133; doi:10.1038/ejhg.2009.123

$\mathrm{T}$ his book is an update of the first edition published in 1999 and results from the author's long experience with Huntington's disease (HD) in a clinical genetic context as well as from his close collaboration with HD association. Most chapters start with a number of key points and many of them are written in a question-answer format that is attractive for the reader. After an introductory chapter with 'Facts and figures about HD', chapter 2 'Physical features of HD' and chapter 3 'Behavioural and emotional aspects of HD' give a clear answer to important practical questions. Moreover, both chapters are well illustrated with many quotes, mainly from partners of patients, but also with a few case studies by professionals. The fourth chapter on juvenile HD does not only answer the questions 'Why use the term JHD? How frequent? Clinical features?' and many other questions, but also looks at the challenges for diagnosis from a parents' perspective as well as a doctor's perspective. 'The genetics of HD' in chapter 5 as well as 'Laboratory testing' in chapter 6 give a considerable amount of important information in a concise way and are well illustrated with clear figures. The glossary at the end of the book is also very useful. 'Genetic counselling for HD' is covered in two separate chapters: chapter 7 about the process involved in a new diagnosis in a family and chapter 8 paying more attention to unaffected family members and to the options for genetic testing that are available for them: predictive testing, prenatal testing and preimplantation genetic diagnosis. Owing to the difficulty of the topic, chapter 9 about 'Changes in the brain' and chapter 10 'What causes selective nerve cell damage?' are slightly less accessible than the previous chapters. Chapter 11 'Current research activities' stresses the importance of research networks as well as the importance of a close collaboration between the HD associations and the research community. The book ends with 'Useful resources and contacts' and information about HD associations in many countries all over the world.

Completely in line with the goal of the series 'thefacts' this book offers interesting and multifaceted information about HD in a clear and accessible manner. It can not only be recommended to families with HD but also to professionals who are involved in the care for HD patients

Gerry Evers-Kiebooms is at the Center for Human Genetics, Psychosocial Genetics Unit University Hospitals, K.U. Leuven, Herestraat 49, Leuven 3000, Belgium. E-mail: Gerry.Kiebooms@med.kuleuven.be

\section{The genomic era and the new frontiers of medicine}

Genomics and clinical medicine

Dhavendra Kumar, Sir David Weatheral

Genomics and Clinical Medicine

ISBN: 978-0-19-518813-4 \$ 175

Published by: Oxford University Press: Oxford, UK: 2008

..........

Gerry Evers-Kiebooms

European Journal of Human Genetics (2010) 18, 133-134; doi:10.1038/ejhg.2009.124

$\mathrm{T}$ the past few years, progress in many fields, including biology, biological engineering, informatics and physics has led from genetics to a wider field, in which the totality of an organism's genes can be considered as a global system in which different components interact with each other and with external factors. The complete genome of Homo sapiens and many other eukaryotic and prokaryotic organisms has been sequenced and stored in public databases. Technological advances have led to many improvements already evident in the results that have been obtained over the past few years from different fields of biological research, such as the different associations between genes and diseases that have emerged from Genome Wide Association Studies.

We are experiencing a real genomics revolution. This is the rationale on which 'Genomics and Clinical Medicine' is based while taking the reader through a survey of the most recent discoveries in genomics and its applications to clinical medicine.

This is a 672 pages multi-author book to which a team of 69 experts from different fields of medical genetics have contributed 
and is edited by Dhavendra Kumar under the advice of Sir David Weatherhall.

The book is structured in 41 chapters grouped into three parts: first, an introductory part in which the general aspects of genomics are explained in nine chapters. Second, a section of 28 chapters in which a general survey of the clinical applications of genomics divided into different disciplines of medicine is provided. Last, a third section of four chapters in which the potential and the impact that genomic applications will have on public health in the coming years, including ethical and legal considerations, are examined.

The general genomics section ranges from a description of the history of genetics and the transition from genetics to genomics to a general description of the genome's known structure and organization, its functional organization, proteomics and epigenomics. The technologies to study genome variations of different orders of magnitude are also considered. In addition, the perspectives of human development and comparative genomics applied to a better understanding of developmental malformations and more, in general, the perspectives of genetic and genomic approaches to the taxonomy of human diseases, are considered. Finally, an overview of nutritional genomics and pharmacogenomics is also given.

The clinical genomics section is a vast survey across different fields of medicine; for each of them, the genetic component is considered and the current state of the art is described. The introductory chapter of this section underlines the importance of bioinformatics on data analysis in different fields of genomics, transcript analysis and proteomics, and the potential of its applications to the current practices of medicine, including diagnostics, therapeutics and prognostics.

The last section is a global view of what the impact of genomics will be on global health. First, through its improvement in developing countries by the control of common monogenic disorders and the control and management of communicable diseases, and second, through its reflection on the diseases of 'Westernization', such as type II diabetes, cardiovascular diseases and stroke. These are all because of the same environmental factors, but reflect different patterns of genetic variability due to varying patterns of evolutionary adaptations in different ethnic groups. A whole chapter is dedicated to the concept of genetic testing and genomic screening, enlisting the criteria that render a genetic test or a genomic screening worthwhile. A chapter is dedicated to ethical, legal and social issues, emphasizing the importance of a valid consent from individuals, in terms of information, understanding, will and competence, all human rights of the patient that are not always easy to respect. Security, confidentiality and data and benefit sharing are all important aspects that have to be considered when saving clinical and biological information in a database.

The last chapter considers the regulation of genomics research, giving an overview of the legal framework in the United Kingdom and United States, concluding that, until now, the law has been confusing and incomplete. We will need new developments in the regulation of medical research to manage the amount of information that is already available, thanks to quick developments in the full respect of human rights.

In conclusion, the book is a nice overview of developments in different fields of genomic medicine, written by a number of experts who give a thorough description of their argument. As the editors themselves recognize, given that each month the amount of information about genomics increases, thanks to advances in technology, bioinformatics and biostatistics, to analyze large amounts of data at the same time, frequent updates will be needed. To use a statement taken from the book itself, the genomic era has just begun

Giuseppe Novelli and Irene Marta Predazzi are at the Department of Biopathology and Diagnostic Imaging, 'Tor Vergata' University of Rome, Roma, Italy. E-mail:novelli@med.uniroma2.it 